PROCEEDINGS OF THE

AMERICAN MATHEMATICAL SOCIETY

Volume 128, Number 9, Pages 2725-2733

S 0002-9939(00)05300-4

Article electronically published on February 28, 2000

\title{
CRITERIA FOR CONVEXITY IN BANACH SPACES
}

\author{
VASSILIS KANELLOPOULOS
}

(Communicated by Dale E. Alspach)

\begin{abstract}
In this paper two convexity criteria are proven. The first one characterizes compact convex sets in a locally convex space and extends a previous result by G. Aumann, while the second one characterizes closed bounded convex sets with the Radon-Nikodým property in a Banach space.
\end{abstract}

\section{INTRODUCTION}

In the present paper the following two theorems are proven:

Theorem 1. Let $K$ be a closed subset of a locally convex space $X$ such that $L=$ $\overline{c o n v} K$ is compact in $X$. The following are equivalent:

(i) $K=L$, that is, $K$ is convex.

(ii) For every closed hyperplane $H$ of $X$ which intersects $K, H \cap K$ is either contractible or has the fixed point property.

Theorem 2. Let $K$ be a weakly closed bounded subset of a Banach space $X$ such that $L=\overline{c o n v} K$ has the Radon-Nikodym property. The following are equivalent:

(i) $K=L$, that is, $K$ is convex.

(ii) For every closed hyperplane $H$ of $X$ which intersects $K, H \cap K$ is either contractible or has the fixed point property in the narrow sense.

(In the above theorems it is assumed that $\operatorname{dim} X \geq 2$.)

A set $A$ is assumed to have the fixed point property in the narrow sense if every continuous compact map of $A$ into itself has a fixed point. It is known that every closed convex subset of a Banach space has that property (Schauder's Theorem, cf. Du1 ) and every compact convex subset of a locally convex space has the fixed point property in the usual sense (Tychonoff's Theorem, cf. Du1). Also, as a direct consequence of the convexity we have that every convex set in a topological vector space is contractible. A closed bounded convex subset $A$ of a Banach space $X$ is assumed to have the Radon-Nikodým property (RNP) if every subset of $A$ has slices of arbitrarily small diameter (for a detailed study of this notion see [B]).

For the case $X=\mathbb{R}^{n}$ similar characterizations have been proven by G. Aumann ([A]) with methods from algebraic topology. Later I. Fàry $([\mathrm{F}]$ ) and A. Kosinski $(\underline{\mathrm{K}})$ gave other proofs for the same result.

Received by the editors October 6, 1997 and, in revised form, October 6, 1998.

2000 Mathematics Subject Classification. Primary 52A07, 46B20; Secondary 46B22.

Key words and phrases. Convex set, locally convex space, Banach space, extreme point, slice, contractible set, Radon-Nikodým property.

This research was financially supported by I.K.Y. (National Foundation for Scholarships).

(C)2000 American Mathematical Society 
The approach of this paper is based on the notion of the $n$-extreme points for Theorem 1 and $n$-denting points of a convex set $K\left(E x_{n}(K)\right.$, resp. $\left.D_{n}(K)\right)$ for Theorem 2 The precise definition of them is given in Section 1 . These are subsets of $K$ strongly related with its extreme points and as is proven in the same section, the sets $\bigcup_{n=0}^{\infty} E x_{n}(K)$ (for compact $K$ ), $\bigcup_{n=0}^{\infty} D_{n}(K)$ (for $K$ with the RNP) are weakly dense in the corresponding set $K$. In Section 2 the $n$-cycles are defined and a sufficient condition is considered for a set $K$ to have a non contractible and without the fixed point property hyperplane section. In Section 3 it is shown that under some assumptions $K$ satisfies the previous condition. Finally in Section 4 the proof of Theorems 1 and 2 is provided, through two more general theorems (Theorems 17 and 18) and some remarks are given.

Notation. The class of all closed subspaces of a locally convex space $X$ which have codimension $m, m \in \mathbb{N}$, is denoted by $\Sigma^{m}(X)$. The class of all affine closed subspaces of $X$ which have codimension $m, m \in \mathbb{N}$, is denoted by $A^{m}(X)$. We also set $\Sigma(X)=\bigcup_{m=0}^{\infty} \Sigma^{m}(X)$ and $A(X)=\bigcup_{m=0}^{\infty} A^{m}(X)$. The members of $A(X)$ will in general be called flats. If $A$ is a subset of a Banach space, then $\mathcal{P}(A)$ denotes the set of all points of continuity of the identity map $I d_{A}:(A$, weak $) \rightarrow(A$, norm $)$.

Some standard notation is also used. If $A \subset X$, then relint $A$ denotes the relative interior of $A, \operatorname{relbd}(A)$ the relative boundary of $A$ and $\operatorname{dim} A$ the $\operatorname{dimension}$ of $A$, that is, the dimension of its affine hull.

\section{SECTION 1}

Let $X$ be a locally convex space, $L \subset X$ be a closed convex set and suppose that $x \in L$. Then $x$ is called an $n$-extreme point of $L(n \in \mathbb{N})$ if $x$ is in the relative interior of no $(n+1)$-dimensional convex subset of $L$. The set of all $n$-extreme points of $L$ is denoted by $E x_{n}(L)$. We also define $E(L)=\bigcup_{n=0}^{\infty} E x_{n}(L)$.

Evidently if $n=0$, we get the usual notion of an extreme point of $L$. Also if $m<n$, then $E x_{m}(L) \subset E x_{n}(L)$. As can easily be proven, the basic property of the $n$-extreme points is the following: A point $x \in E x_{n}(L)$ if and only if there exists a unique face $A_{x}$ of $L$ which contains $x$ in its relative interior and with $\operatorname{dim} A_{x} \leq n$. (As usually denoted a face of $L$ is a closed convex set $A \subset L$ such that each segment $\left[x_{1}, x_{2}\right] \subset L$ with $A \cap$ relint $\left[x_{1}, x_{2}\right] \neq \emptyset$ is contained in $A$.) The reader can refer to $[\underline{\mathrm{S}}]$ for a more extensive study of the notion of $n$-extreme points in finite dimensional spaces.

At this point let us give some examples for the $n$-extreme points:

(a) If $X=\ell^{p}, 1<p<\infty, L=B_{\ell^{p}}$ (the unit ball of $\ell^{p}$ ), then it can be shown that $E x_{0}(L)=E x_{n}(L)=S_{\ell^{p}}$ (the unit sphere of $\ell^{p}$ ), for every $n \in \mathbb{N}$.

(b) If $X=\ell^{1}, L=B_{\ell^{1}}$, then we have that:

$$
E x_{n-1}(L)=\left\{\lambda_{m_{1}} e_{m_{1}}+\ldots+\lambda_{m_{n}} e_{m_{n}}: m_{1}<\ldots<m_{n}, \quad \sum_{i=1}^{n}\left|\lambda_{m_{i}}\right|=1\right\}
$$

for every $n \geq 1$, where $\left\{e_{1}, e_{2}, \ldots\right\}$ is the usual basis of $\ell^{1}$.

(c) If $X=c_{0}$ and $L=B_{c_{0}}$, then $E x_{n}(L)=\emptyset$ for every $n \geq 0$.

Lemma 3. Let $L$ be a closed convex subset of $X$ and suppose that a flat $F \in$ $A^{m}(X)$ intersects $L$. Then $F \cap L$ is a closed convex subset of $X$ such that $E x_{0}(F \cap L)$ $\subset E x_{m}(L)$. 
Proof. If $E x_{0}(F \cap L)=\emptyset$, then the lemma trivially holds. So let us assume that $E x_{0}(F \cap L) \neq \emptyset$ and $z \in E x_{0}(F \cap L)$.

Suppose that $z \notin E x_{m}(L)$. Then there exists an $(m+1)$-dimensional convex set $K$ with $z \in$ relint $K$ such that $K \subset L$. Since $F$ is a flat of codimension $m$ and $z \in F$ is at the same time in the relative interior of an $(m+1)$-dimensional convex set, there exists a segment $\left[x_{1}, x_{2}\right] \subset F \cap K \subset F \cap L$, such that $z$ is the midpoint of $\left[x_{1}, x_{2}\right]$. Hence $z \notin E x_{0}(F \cap L)$, which is a contradiction.

Proposition 4. If $L$ is a compact convex subset of a locally convex space $X$, then $L=\overline{E(L)}$.

Proof. If $L$ is finite dimensional, then evidently $L=E(L)=E x_{m}(L)$ where $m=$ $\operatorname{dim} L$. If $L$ is infinite dimensional, we can assume that $L$ is weakly compact and so it is enough to prove that $E(L)$ is weakly dense in $L$.

Let $V$ be a weakly open basic neighborhood of a point $x_{0} \in L$. Since $E(L)=$ $\bigcup_{n=0}^{\infty} E x_{n}(L)$, it suffices to prove that there exists an $m \in \mathbb{N}$ which depends on $V$ such that $V \cap E x_{m}(L) \neq \emptyset$. Suppose that $V$ is determined by the functionals $f_{1}, \ldots, f_{k} \in X^{*}$. We set $F=x_{0}+\bigcap_{i=1}^{k} \operatorname{Kerf} f_{i}$. Then $F \in A^{m}(X)$ for some $m \leq k$, $x_{0} \in F$ and $F \subset V$. Evidently $F \cap L$ is a non empty compact convex subset of $X$ and so $E x_{0}(F \cap L) \neq \emptyset$ by Krein-Milman's Theorem. According to the previous lemma $E x_{0}(F \cap L) \subset E x_{m}(L)$, hence $\emptyset \neq E x_{0}(F \cap L) \subset F \cap E x_{m}(L) \subset$ $V \cap \operatorname{Ex}(L)$.

Remark 1. Using the same method as in the previous proposition, one can prove that if $L$ is a closed convex subset of a locally convex space such that $L$ has the Krein-Milman Property (that is, each closed convex subset of $L$ has an extreme point), then $L=\overline{E(L)}^{w}$.

Let us suppose for the rest of Section 1 that $X$ is a Banach space. Let $L$ be a closed bounded convex subset of $X$. A point $x \in L$ is an $n$-denting point of $L$ $(n \in \mathbb{N})$ if: (a) $x$ is an $n$-extreme point of $L$ and (b) $x$ is a point of continuity of the identity map $i d_{L}:(L$, weak $) \rightarrow(L$, norm $)$, that is, $x \in E x_{n}(L) \cap \mathcal{P}(L)$. The set of all $n$-denting points of $L$ is denoted by $D_{n}(L)$. We also define $D(L)=$ $\bigcup_{n=0}^{\infty} D_{n}(L)$.

Evidently if $n=0$, we get the usual notion of a denting point of $L$. Also if $m<n$, then $D_{m}(L) \subset D_{n}(L)$. Since every $x \in D_{n}(L)$ is an $n$-extreme point of $L$, there exists a unique face $A_{x}$ of $L$ which contains $x$ in its relative interior and with $\operatorname{dim} A_{x} \leq n$. The converse does not hold in general, but if $L$ is finitedimensional, then evidently $E x_{n}(L)=D_{n}(L)$ for every $n \in \mathbb{N}$, since the weak and norm topologies coincide on $L$. The examples (a) and (b) of $n$-extreme points, given in the beginning of this section, are also examples for $n$-denting points.

The following result was suggested by Professor S. A. Argyros.

Proposition 5. Let $L$ be a bounded convex subset of a Banach space $X$ and suppose that $V$ is a weakly open basic neighborhood of a point $x_{0} \in L$. Then $\mathcal{P}(\bar{V} \cap L) \subset$ $\mathcal{P}(L)$.

Proof. Evidently if $\mathcal{P}(\bar{V} \cap L)=\emptyset$, the theorem trivially holds. So let us suppose that $z_{0} \in \mathcal{P}(\bar{V} \cap L)$. Then for every $\varepsilon>0$ there exists a weakly open basic neighborhood of $z_{0}$ which is denoted by $W_{\varepsilon}$ such that $\operatorname{diam}\left(W_{\varepsilon} \cap \bar{V} \cap L\right)<\varepsilon$. If $z_{0} \in V$, then obviously $W_{\varepsilon} \cap V$ is a weakly open neighborhood of $z_{0}$ such that $\operatorname{diam}\left(W_{\varepsilon} \cap V \cap L\right)<\varepsilon$. Since $\varepsilon$ is arbitrarily chosen, $z_{0} \in \mathcal{P}(L)$. 
If $z_{0} \in \bar{V} \backslash V$, then without loss of generality we may suppose the following:

(1) $x_{0}=0$ and $V=\left\{x \in X:\left|f_{i}(x)\right|<1\right.$ for $\left.i=1, \ldots, k\right\}$ where $f_{1}, \ldots, f_{k} \in X^{*}$ and so $\left|f_{i}\left(z_{0}\right)\right| \leq 1$ for $i=1, \ldots, k$.

(2) $0 \notin W_{\varepsilon}$ and $W_{\varepsilon}=\left\{x \in X:\left|g_{j}\left(x-z_{0}\right)\right|<1\right.$ for $\left.j=1, \ldots, m\right\}$ where $g_{1}, \ldots, g_{m}$ $\in X^{*}$. Hence $\left|g_{j}\left(z_{0}\right)\right| \geq 1$, for some $j \in\{1, \ldots, m\}$.

(3) $0<\varepsilon<\frac{M}{C}$ where $M=\sup \{\|x\|: x \in L\}$ and $C=\max \left\{\mid g_{j}\left(z_{0}\right): 1 \leq j \leq m\right\}$. According to (2) it is clear that $C \geq 1$ and $M>0$.

Let us define:

$V^{\prime}=\left\{x \in X:\left|f_{i}(x)\right|<1+\frac{\varepsilon}{2 M}\right.$ for $\left.i=1, \ldots, k\right\}$ and

$W_{\varepsilon}^{\prime}=\left\{x \in X:\left|g_{j}\left(x-z_{0}\right)\right|<1-\frac{\varepsilon C}{M}\right.$ for $\left.j=1, \ldots, m\right\}$.

Claim. (a) Let $r \in(0,1)$ such that $\frac{1}{1+\frac{\varepsilon}{M}}<r<\frac{1}{1+\frac{\varepsilon}{2 M}}$. Then $r\left(V^{\prime} \cap W_{\varepsilon}^{\prime} \cap L\right) \subset$ $V \cap W_{\varepsilon} \cap L$.

(b) $\operatorname{diam}\left(V^{\prime} \cap W_{\varepsilon}^{\prime} \cap L\right)<4 \varepsilon$.

Proof of the Claim. (a) Let $x \in V^{\prime} \cap W_{\varepsilon}^{\prime} \cap L$. Then

(1) For $i=1, \ldots, k\left|f_{i}(r x)\right|=r\left|f_{i}(x)\right|<r\left(1+\frac{\varepsilon}{2 M}\right)<1$. Hence $r x \in V$.

(2) For $j=1, \ldots, m$,

$$
\begin{aligned}
& \left|g_{j}\left(r x-z_{0}\right)\right|=\left|g_{j}\left(r x-r z_{0}+r z_{0}-z_{0}\right)\right| \leq r\left|g_{j}\left(x-z_{0}\right)\right|+(1-r)\left|g_{j}\left(z_{0}\right)\right| \\
& \quad<r\left(1-\frac{\varepsilon C}{M}\right)+(1-r) C=r-r\left(\frac{\varepsilon}{M}+1\right) C+C<r-C+C=r<1 .
\end{aligned}
$$

Hence for $j=1, \ldots, m\left|g_{j}\left(r x-z_{0}\right)\right|<1$ and so $r x \in W_{\varepsilon}$.

(3) Since $L$ is convex and $0 \in L$, then $(1-r) 0+r x=r x \in L$.

By (1), (2) and (3) $r x \in V \cap W_{\varepsilon} \cap L$ for every $x \in V^{\prime} \cap W_{\varepsilon}^{\prime} \cap L$.

(b) Let $x \in V^{\prime} \cap W_{\varepsilon}^{\prime} \cap L$ and $r \in(0,1)$ as in (a). Then $\|r x-x\|=(1-r)\|x\|<$ $\left(1-\frac{1}{1+\frac{\varepsilon}{M}}\right) M=\frac{\varepsilon}{1+\frac{\varepsilon}{M}}$ and $\left\|r x-z_{0}\right\|<\varepsilon$ since $z_{0}, r x \in\left(\bar{V} \cap W_{\varepsilon} \cap L\right)$ (by (a)) and $\operatorname{diam}\left(\bar{V} \cap W_{\varepsilon} \cap L\right)<\varepsilon$. Therefore $\left\|x-z_{0}\right\| \leq\|r x-x\|+\left\|r x-z_{0}\right\|<\varepsilon+\frac{\varepsilon}{1+\frac{\varepsilon}{M}}$.

Hence $\sup \left\{\|x-y\|: x, y \in V^{\prime} \cap W_{\varepsilon}^{\prime} \cap L\right\}<4 \varepsilon$. The proof of the claim is complete.

Since $V^{\prime} \cap W_{\varepsilon}^{\prime}$ is evidently a weakly open neighborhood of $z_{0}$ and $\varepsilon$ is arbitrarily chosen, we conclude by (b) of the claim that $z_{0} \in \mathcal{P}(L)$ and thus the proof of Proposition 5 is complete.

Lemma 6. Let $L$ be a closed bounded convex subset of a Banach space $X$ and suppose that $V$ is a weakly open basic neighborhood of a point $x_{0} \in L$. Then $\bar{V} \cap L$ is a closed bounded convex subset of $X$ with $E x_{0}(\bar{V} \cap L) \subset E(L)$.

Proof. It is evident that the set $\bar{V} \cap L$ is a closed bounded convex subset of $X$. If $E x_{0}(\bar{V} \cap L)=\emptyset$, then the lemma trivially holds. So let us suppose that $z \in$ $E x_{0}(\bar{V} \cap L)$. Following the proof of Proposition 4, there exists an $F \in A^{m}(X)$ for some $m \in \mathbb{N}$ such that $z \in F$ and $F \subset \bar{V}$. Obviously $z \in E x_{0}(F \cap L)$ and by Lemma 3, $z \in E x_{m}(L)$. Therefore $z \in E(L)$.

Proposition 7. Let $L$ be a closed bounded convex subset of a Banach space $X$ and suppose that $L$ has the RNP. Then $L=\overline{D(L)}^{w}$.

Proof. Since $L$ is a norm closed convex subset of $X, L$ is weakly closed as well. So it is enough to prove that $D(L)$ is weakly dense in $L$. To this end, let us assume that $V$ is a weakly open basic neighborhood of a point $x_{0} \in L$. It is clear that there exists a 
weakly open basic neighborhood $V^{\prime}$ of the same point such that $\overline{V^{\prime}} \subset V$. Evidently $\overline{V^{\prime}} \cap L$ is a closed convex subset of $L$ and since $L$ has the RNP, $D_{0}\left(\overline{V^{\prime}} \cap L\right) \neq \emptyset$ by the Phelps-Bourgain Theorem (cf. [B]). By Proposition 5 and Lemma 6 we have that $D_{0}\left(\overline{V^{\prime}} \cap L\right) \subset E(L) \cap \mathcal{P}(L)=D(L)$. Hence $\emptyset \neq \overline{V^{\prime}} \cap D(L) \subset V \cap D(L)$.

\section{SECTION 2}

Let $S, F$ be closed subspaces of a locally convex space $X$ where $F$ is finite dimensional and $S \cap F=\{0\}$. We write $S \oplus F$ for the linear span of $S \cup F$. It is obvious that every $x \in S \oplus F$ can be uniquely written as $x=s+f$, where $s \in S$ and $f \in F$. Hence we can define the projection $P_{F}: S \oplus F \rightarrow F$, where $P_{F}(x)=f$, if $x=s+f$. In the trivial case where $S=\{0\}, P_{F}$ is the identity map on $F$. Since $X$ is a locally convex space and $F$ is finite dimensional, it can be proven that $S \oplus F$ is a closed subspace of $X$ and the projection $P_{F}$ is continuous.

Notation. The relative boundary of an $(n+1)$-dimensional compact convex set will be called an $n$-cycle. We denote by $\mathcal{K}_{0}^{n}$ the class of all $n$-dimensional compact convex subsets of $X$ which contain 0 in their relative interior. The relative boundary of a set $L \in \mathcal{K}_{0}^{n+1}$ is called an $n$-cycle around 0 .

Lemma 8. Let $K$ be a subset of a locally space $X$ and $H \in \Sigma^{1}(X)$ with the following properties:

(i) $H=F \oplus F_{n}$, where $F \in \Sigma^{n+1}(X), F_{n}$ is an $n$-dimensional subspace of $X$ and $n \geq 1$.

(ii) $F \cap K=\emptyset$.

(iii) $F_{n} \cap K$ contains an $(n-1)$-cycle $C$ around 0 .

Then $C$ is a retract of $H \cap K$.

Proof. For every $x \in F_{n} \backslash\{0\}$ we define $P_{C}(x)=C \cap\{\lambda x: \lambda>0\}$. Since $C$ is an $(n-1)$-cycle, $P_{C}$ is well defined and continuous. Let $P_{F_{n}}: H \rightarrow F_{n}$ be the projection onto $F_{n}$. We note that $P_{F_{n}}(H \cap K) \subset F_{n} \backslash\{0\}$, since $P_{F_{n}}(x)=0$ if and only if $x \in F$, whereas $F \cap K=\emptyset$ by assumption (ii).

So the map $S: H \cap K \rightarrow C$ defined by $S(x)=P_{C}\left(P_{F_{n}}(x)\right)$, where $x \in H \cap K$, is a well defined continuous map onto $C$ leaving every point of $C$ fixed. That is, $C$ is a retract of $H \cap K$.

Remark 2. Suppose that a set $A \subset X$ is contractible or has the fixed point property in the usual or in the narrow sense. Then there is no $n$-cycle which is a retract of $A$. Indeed, it is easy to see that every retract of $A$ has the above mentioned properties, whereas an $n$-cycle as an homeomorphic image of the unit sphere $S^{n}$ of $\mathbb{R}^{n+1}$, neither is contractible by Brouwer's Theorem (cf. [Du1]) nor has the fixed point property in the usual or in the narrow sense.

\section{SECTION 3}

In the following and up to Proposition 11 let us denote by $X$ a locally convex space $X$ and by $K$ a closed subset of $X$ such that the set $L=\overline{c o n v} K$ is compact.

Let us define $E x_{m}^{*}(L)=E x_{m}(L) \backslash E x_{m-1}(L), m \geq 1$, and suppose that $0 \in$ $E x_{m}^{*}(L)$. According to the basic property of the $n$-extreme points (Section 1), there exists a unique face $A_{0}$ of $L$ which contains 0 in its relative interior. Let us denote by $F_{m}$ the linear span of $A_{0}$. It is obvious that $A_{0} \in \mathcal{K}_{0}^{m}$ and so $F_{m}$ is an 
$m$-dimensional (and thus closed) subspace of $X$. Since $F_{m}$ is finite dimensional, there exists a $Y \in \Sigma^{m}(X)$ such that $Y \oplus F_{m}=X$.

In the following Lemma 9 we shall need Choquet's Lemma (cf. [HHZ]), that is, in every compact convex subset $L$ of $X$ the slices of $L$ consist of a neighborhood base for the extreme points of $L$. As is known, a slice of $L$ is the intersection of $L$ with an open halfspace of $X$. Formally, if $f \in X^{*}$ and $a \in \mathbb{R}$, then a slice of $L$ can be written as $S(f, L, a)=\{y \in L: f(y)>a\}$.

Lemma 9. Let $K, L, A_{0}, F_{m}, Y$ be as above and $0 \in E x_{m}^{*}(L) \backslash K, 1 \leq m<\operatorname{dim} X$. Then:

(a) $0 \in E x_{0}(Y \cap L)$.

(b) There exist an $H \in \Sigma^{1}(X)$ and an $F \in \Sigma^{m+1}(X), F \subset Y$ such that:

(i) $H=F \oplus F_{m}$

(ii) $F \cap K=\emptyset$.

Proof. (a) Obviously $Y \cap L$ is a compact convex subset of $L$ containing 0 . If $0 \notin$ $E x_{0}(Y \cap L)$, then there exists a closed segment $\left[x_{1}, x_{2}\right] \subset Y \cap L$ with midpoint at 0 and $x_{1} \neq x_{2}$. Since $A_{0}$ is a face of $L$ and $0 \in \operatorname{relint}\left[x_{1}, x_{2}\right] \cap A_{0}$, we have that $\left[x_{1}, x_{2}\right] \subset A_{0}$. Hence $\left[x_{1}, x_{2}\right] \subset Y \cap L \cap A_{0} \subset Y \cap L \cap F_{m}=\{0\}$ which is a contradiction.

(b) We notice that $\operatorname{dim} Y \geq 1$ since $m<\operatorname{dim} X$. Two cases are distinguished. Either $Y \cap K=\emptyset$ or $Y \cap K \neq \emptyset$.

If $Y \cap K=\emptyset$, then for every $F \in \Sigma^{1}(Y)$ we have $F \in \Sigma^{m+1}(X), F \oplus F_{m}=$ $H \in \Sigma^{1}(X)$ and $F \cap K \subset Y \cap K=\emptyset$. (If $\operatorname{dim} Y=1$, then $F$ is necessarily the trivial space $\{0\}$. )

If $Y \cap K \neq \emptyset$, then since $0 \notin K$ and $K$ is closed, there exists an open neighborhood $V$ of 0 in $X$ such that $V \cap K=\emptyset$. But then $V \cap(Y \cap L)$ is an open neighborhood of 0 in the compact convex set $Y \cap L$. Since (by (a)) $0 \in E x_{0}(Y \cap L)$ Choquet's lemma can be applied. Therefore there exists a slice $S=S(f, Y \cap L, a)$ such that $0 \in S$ and $S \subset V \cap(Y \cap L)$. Hence, $S \cap K=\emptyset$.

Let $G=K e r f$. Then $G \in \Sigma^{1}(X)$ and since $0 \in S, G \cap(Y \cap L) \subset S$. We note that $Y$ is not contained in $G$, since otherwise $Y \cap K=(G \cap Y) \cap(L \cap K)=$ $G \cap(Y \cap L) \cap K \subset S \cap K=\emptyset$, which is a contradiction to our assumption where $Y \cap K \neq \emptyset$.

Let $F=G \cap Y$. Since $Y \in \Sigma^{m}(X), G \in \Sigma^{1}(X)$ and $Y \nsubseteq G$ we conclude that $F \in \Sigma^{m+1}(X)$. We also have that $F \cap F_{m}=\{0\}$, since $F \subset Y$, and so the subspace $H=F \oplus F_{m}$ is a well defined closed hyperplane of $X$. Finally $F \cap K=$ $(G \cap Y) \cap(L \cap K)=G \cap(Y \cap L) \cap K \subset S \cap K=\emptyset$.

Lemma 10. Let $0 \in E x_{m}(L), m \geq 1$ and $E x_{m-1}(L) \subset K$. Then relbd $A_{0} \subset K$ and therefore $F_{m} \cap K$ contains an $(m-1)$-cycle around 0 .

Proof. Since $A_{0} \in \mathcal{K}_{0}^{m}, \operatorname{relbd} A_{0}$ is an $(m-1)$-cycle around 0 and $\operatorname{relbd} A_{0}=$ $E x_{m-1}\left(A_{0}\right)$. On the other hand $A_{0}$ is a face of $L$. Hence every face of $A_{0}$ is a face of $L$ as well. By the basic property of $m$-extreme points (as it is stated in Section 1), this means that $E x_{m-1}\left(A_{0}\right) \subset E x_{m-1}(L)$. Since $E x_{m-1}(L) \subset K$, relbd $A_{0} \subset K$ as well.

Proposition 11. Let $K$ be a closed subset of a locally convex space $X$ with the following properties:

(i) $\quad L=\overline{c o n v} K$ is a compact subset of $X$. 
(ii) $\quad E x_{m-1}(L) \subset K$, for some $m \geq 1$.

(iii) $0 \in E x_{m}(L) \backslash K$.

Then there exist a hyperplane $H \in \Sigma^{1}(X)$ and an $n$-cycle $C$ around 0 (where $n=m-1$ if $m<\operatorname{dim} X$ or $n=m-2$ if $\operatorname{dim} X=m)$, such that $C$ is a retract of $H \cap K$.

Proof. Obviously $0 \in E x_{m}^{*}(L) \backslash K$. Two cases are distinguished:

(1) $1 \leq m<\operatorname{dim} X$.

By Lemmas 9 and 10 there exist an $H \in \Sigma^{1}(X)$ and an $F \in \Sigma^{m+1}(X)$ such that: (i) $H=F \oplus F_{m}$ and $F_{m}$ is an $m$-dimensional subspace of $X$; (ii) $F \cap K=\emptyset$; (iii) $F_{m} \cap K$ contains a $(m-1)$-cycle $C$ around 0. By Lemma 8, $C$ is a retract of $H \cap K$.

(2) $m=\operatorname{dim} X \geq 2$.

Then $F_{m}=X, X$ is isomorphic to $\mathbb{R}^{m}$ and $A_{0}=L$. Let us denote by $B d L$ the boundary of $L$. By Lemma 10, $B d L \subset K$. For every $H \in \Sigma^{1}(X)$ the following trivially hold: (i) $H=\{0\} \oplus H$ and $H$ is an $(m-1)$-dimensional subspace of $X$; (ii) $\{0\} \cap K=\emptyset$; (iii) $H \cap K$ contains an $(m-2)$-cycle $C$ around 0 , where $C=B d L \cap H$. By Lemma 8 for each $H \in \Sigma^{1}(X)$ there exists an $(m-2)$-cycle around 0 which is a retract of $H \cap K$.

Hereafter let us denote by $X$ a Banach space.

Lemma 12. Let $L$ be a convex subset of a Banach space $X$ and suppose that $z \in$ $\mathcal{P}(L)$. If $z=r z_{1}+(1-r) z_{2}$, where $r \in(0,1)$, and $z_{1}, z_{2} \in L$, then $z_{1}, z_{2} \in \mathcal{P}(L)$ as well.

Proof. We prove that $z_{1} \in \mathcal{P}(L)$ (the proof of $z_{2}$ is similar). Since $z \in \mathcal{P}(L)$, for every $\varepsilon>0$ there exists a weakly open neighborhood $V_{\varepsilon}$ of $z$ such that $\operatorname{diam}\left(V_{\varepsilon} \cap L\right)<$ $\varepsilon$. We define $V_{\varepsilon}^{1}=\frac{1}{r}\left(V_{\varepsilon}-(1-r) z_{2}\right)$. Evidently, $V_{\varepsilon}^{1}$ is a weakly open neighborhood of $z_{1}$. It is easy to see that $\operatorname{diam}\left(V_{\varepsilon}^{1} \cap L\right) \leq \frac{1}{r} \varepsilon$. As $\varepsilon$ is arbitrarily chosen, it follows that $z_{1} \in \mathcal{P}(L)$.

The lemma corresponding to Choquet's Lemma in the case of 0-denting points, due to Troyanski, Lin and Lin (cf. [HHZ], Chapter 5), is the following:

Lemma 13. Let $L$ be a closed bounded convex subset of a Banach space $X$ and suppose that $z \in D_{0}(L)$. Then the slices of $L$ consist a neighborhood base for the norm topology at $z$.

Let us suppose in the sequel that $K$ is a closed bounded subset of $X$ and $L=$ $\overline{c o n v} K$. Let us also define $D_{m}^{*}(L)=D_{m}(L) \backslash D_{m-1}(L)$ for $m \geq 1$ and suppose that $0 \in D_{m}^{*}(L)$. Hence $0 \in E x_{m}^{*}(L)$ as well and so we can define $A_{0}, F_{m}$ and $Y$ just as we have defined them in the beginning of Section 3.

The proof of the following lemma is similar to that of Lemma 9] except that $Y \cap L$ is a closed bounded subset of a Banach space $X, 0 \in D_{0}(Y \cap L)$ and we apply Lemma 13 instead of Choquet's Lemma.

Lemma 14. Let $K, L, A_{0}, F_{m}, Y$ be as above and $0 \in D_{m}^{*}(L) \backslash K, 1 \leq m<\operatorname{dim} X$. Then:

(a) $0 \in D_{0}(Y \cap L)$.

(b) There exist an $H \in \Sigma^{1}(X)$ and an $F \in \Sigma^{m+1}(X), F \subset Y$ such that:

(i) $H=F \oplus F_{m}$.

(ii) $F \cap K=\emptyset$. 
Lemma 15. Let $0 \in D_{m}(L), m \geq 1$, and $D_{m-1}(L) \subset K$. Then relbd $A_{0} \subset K$ and therefore $F_{m} \cap K$ contains an $(m-1)$-cycle around 0 .

Proof. Using the arguments of Lemma 10, it can be shown that

$$
\operatorname{relbd} A_{0}=E x_{m-1}\left(A_{0}\right) \subset E x_{m-1}(L) .
$$

Since $0 \in$ relint $A_{0}$, for every point $z_{1} \in \operatorname{relbd} A_{0}$, there exists another point $z_{2} \in$ relbd $A_{0}$ such that $0=r z_{1}+(1-r) z_{2}$ for some $r \in(0,1)$. By Lemma 12, since $0 \in \mathcal{P}(L)$ we have that $z_{1}, z_{2} \in \mathcal{P}(L)$. Hence relbd $A_{0} \subset E x_{m-1}(L) \cap \mathcal{P}(L)=$ $D_{m-1}(L)$. Since $D_{m-1}(L) \subset K$, relbd $A_{0} \subset K$ as well.

Proposition 16. Let $K$ be a closed bounded subset of a Banach space $X$ and $L=$ $\overline{\mathrm{conv}} K$, with the following properties:

(i) $D_{m-1}(L) \subset K$, for some $m \geq 1$.

(ii) $0 \in D_{m}(L) \backslash K$.

Then there exist a hyperplane $H \in \Sigma^{1}(X)$ and an $n$-cycle $C$ around 0 (where $n=m-1$ if $m<\operatorname{dim} X$ or $n=m-2$ if $\operatorname{dim} X=m$ ), such that $C$ is a retract of $H \cap K$.

Proof. Similar to that of Proposition 11 except that now we use Lemmas 14 and 15 instead of Lemmas 9 and 10.

\section{SECTION 4}

(a) Proof of Theorem 1. (i) $\Rightarrow$ (ii) If $K$ is a compact convex subset of $X$ and $H \in \Sigma^{1}(X)$ such that $H \cap K \neq \emptyset$, then evidently $H \cap K$ is a non empty compact convex subset of $X$ and therefore it is contractible and has the fixed point property, as is noted in the Introduction.

(ii) $\Rightarrow$ (i) According to Remark 2 it is enough to prove the following:

Theorem 17. Let $K$ be a closed subset of a locally convex space $X$ such that $L=$ $\overline{c o n v} K$ is compact in $X$ and suppose that for every closed hyperplane $H$ of $X$ which intersects $K$, there is no $n$-cycle, $n \in \mathbb{N}$, which is a retract of $H \cap K$. Then $E(L) \subset K$ and $K$ is convex.

Proof. We will prove inductively that $E x_{m}(L) \subset K$, for every $m \in \mathbb{N}$. For $m=0$ it is a consequence of Choquet's Lemma. Let us suppose that for some $m \geq 1$, $E x_{m-1}(L) \subset K$ and $E x_{m}(L) \nsubseteq K$. Then there exists a point $x_{0} \in E x_{m}(L) \backslash K$ which without loss of generality is the point 0 . But then by Proposition 11 there exist an $H \in \Sigma^{1}(X)$ and an $n$-cycle $C$ such that $C$ is a retract of $H \cap K$, a contradiction. Hence $E x_{m}(L) \subset K$ for every $m \in \mathbb{N}$ and so $E(L) \subset K$. Since by Proposition $4=\overline{E(L)}$, we have that $K=L$ and so $K$ is convex.

The proof of Theorem 1 is complete.

(b) Proof of Theorem [ (i) $\Rightarrow$ (ii) It is similar to that of Theorem 1 except that we use Schauder's fixed point theorem in place of Tychonoff's.

(ii) $\Rightarrow$ (i) Like in Theorem 1 it is enough to prove the following:

Theorem 18. Let $K$ be a closed bounded subset of a Banach space $X$ such that $L=\overline{c o n v} K$ has the RNP and suppose that for every closed hyperplane $H$ of $X$ which intersects $K$, there is no n-cycle which is a retract of $H \cap K$. Then $D(L) \subset K$ and $K$ is weakly dense in $L$. 
Proof. Similar to that of the Theorem [17] except that we use Lemma [13. Proposition [16 and Proposition[7] instead of Choquet's Lemma, Proposition 11] and Proposition 4 respectively.

The proof of Theorem 2 is complete.

Remark 3. In Theorem 18 the conclusion that $K$ is weakly dense in $L$ cannot be improved. For example let $X=\ell^{2}$ and $K=S_{\ell^{2}}$; then $L=\overline{c o n v} K=B_{\ell^{2}}$ and as is known $B_{\ell^{2}}$ has the RNP. If $H$ is a closed hyperplane of $\ell^{2}$ that intersects $S_{\ell^{2}}$, then $H \cap S_{\ell^{2}}$ is contractible and has the fixed point property in the narrow sense. Indeed $H \cap S_{\ell^{2}}$ is a single point or a retract of $H \cap B_{\ell^{2}}$, as follows by the related theorem of Dugundji (Du2]). Of course $S_{\ell^{2}}$ is not convex but ${\overline{S_{\ell^{2}}}}^{w}=B_{\ell^{2}}$.

Remark 4. Let us denote by $(X, \tau)$ a Banach space $X$ equipped with a topology $\tau$ where $\tau$ is the norm or the weak or the weak* topology (if $X$ is a dual space). Then $(X, \tau)$ is a locally convex space. Various theorems (such as Krein's theorem if $\tau$ is the weak topology) establish that if $K$ is a compact subset of $(X, \tau)$, then $L=\overline{\operatorname{conv}} K$ (where the closure is taken with respect to the topology $\tau$ ) is compact as well. So, as a direct consequence of Theorem 1, we have the following:

Theorem 19. Let $K$ be a compact subset of $(X, \tau)$. Then $K$ is convex, if and only if for every closed hyperplane $H$ of $X$ which intersects $K, H \cap K$ is either contractible or has the fixed point property.

\section{ACKNOWLEDGMENTS}

I would like to thank Professor S. P. Zervos who brought to my attention questions related to this subject. I also thank Professor S. A. Argyros and V. Felouzis for their valuable help during the preparation of this paper.

\section{REFERENCES}

[A] G.Aumann On a topological characterization of compact convex point sets Ann. of Math 37, (1936) pp. 443-447.

[B] R. Bourgin Geometric aspects of convex sets with the Radon-Nikodým property Lecture notes in Math. Springer-Verlag 1983. MR 85d:46023

[Du1] J. Dugundji Topology Allyn and Bacon Boston 1966. MR 33:1824

[Du2] J. Dugundji An extension of Tietze's Theorem. Pac. J. Math.1 (1951) pp. 353-367. MR 13:373c

[F] I. Fàry A characterization of convex bodies Am. Math. Monthly, vol 69, (1962) pp. 25-31. [HHZ] P. Habala-P. Hajek-V. Zizler Introduction to Banach spaces Matfyz. Press 1996.

[K] A. Kosinski Note on star shaped sets Proc. Amer. Math. Soc. 13, (1962) 931-933. MR 26:669

[S] R. Schneider Convex bodies. The Brun-Minkowski Theory. Cambridge University Press 1993. MR 94d:52007

Department of Mathematics, University of Athens, Panepistimiopolis 15784, Athens, GREECE

E-mail address: bkanel@math.uoa.gr 